American Journal of Surgical Research and Reviews
(ISSN:2637-5087)

\title{
Modern Diagnosis and Surgical Management of Thoracic Outlet Syndrome: A Comprehensive Review
}

\section{Farid Gharagozloo*, M.D., Nabhan Atiquzzaman, Mark Meyer, M.D., Scott Werden M.D.}

Center for Advanced Thoracic Surgery. Global Robotics Institute. Advent health Celebration. University of Central Florida.

\begin{abstract}
Conventionally TOS has been thought to represent a group of ${ }^{*}$ Correspondence to Author: diverse disorders that result in compression of the neurovascular Farid Gharagozloo, M.D. bundle exiting the thoracic outlet. Until recently, TOS classification Center for Advanced thoracic has been based on symptoms, rather than the underlying Surgery. 400 celebration Place, pathology, with the subgroups consisting of neurogenic (NTOS), Celebration, Florida, 34786. venous (VTOS or PSS), and arterial (ATOS). Neurogenic TOS Phone: 407-303-3827 accounts for over $95 \%$ of the cases, followed by venous (3-5\%) and arterial (1-2\%). Neurogenic TOS (NTOS) has been further divided into True NTOS (TNTOS) and Disputed NTOS (DNTOS), with DNTOS reportedly representing $95-99 \%$ of all neurogenic cases. In order to decrease confusion and to improve therapeutic results with TOS, the disease should be classified based on the underlying pathologic entity. Acquired and traumatic abnormalities of the clavicle and first rib should be classified separately. Clearly after the more common and objectively supported diagnoses of conditions that result in neurovascular symptoms of the upper extremity, such as cervical spine disease, carpal tunnel disease, and nerve entrapment syndromes, have been ruled out, there remains a group of patients who are suspected of having TOS. In these patients, rather than the more usual classification such as arterial, venous, or neurogenic, the more accurate approach from a diagnostic and therapeutic approach is to classify them as: Cervical Rib Disease: Patients with cervical rib syndrome (CRS) can have complications relating to compression of the subclavian artery (previously referred to as ATOS) and the brachial plexus(previously referred to as True NTOS) secondary

Authors have no competing interest.

How to cite this article:

Farid Gharagozloo, Nabhan Atiquzzaman, Mark Meyer, Scott Werden. Modern Diagnosis and Surgical Management of Thoracic Outlet Syndrome: A Comprehensive Review. American Journal of Surgical Research and Reviews, 2021; 4:27.

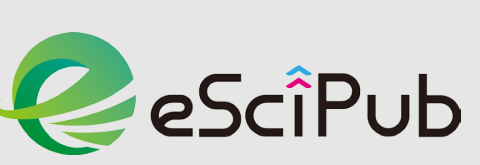
eSciPub LLC, Houston, TX USA. Website: https://escipub.com/ By using the site/services, you are agreeing to our Policies: https:// escipub.com/terms-privacy-policydisclaimer/
\end{abstract}


to a well-formed cervical rib, or to an incompletely formed first rib, fibrous band associated with a rudimentary cervical rib, or a giant transverse process of C7. Thoracic Outlet Disease or "Subclavian Vein Compression Syndrome": In these patients an abnormal first rib at its junction with the sternum results in compression of the subclavian vein at the subclavian-innominate junction. Compression of the vein results in venous hypertension in the upper extremity and resultant neurologic symptoms. With prolonged compression of the subclavian-innominate junction, the vein clots giving rise to Paget-Schroetter syndrome. Therefore, patients who have been previously classified as Disputed Neurogenic and Venous TOS represent a variable symptomatic presentation of the same pathologic entity, which affects the subclavian vein. Presently MRA of the thoracic outlet with arm maneuvers is the test of choice in patients suspected of having TOS. This test shows the abnormal bony tubercle on the first rib with extrinsic compression of the subclavian innominate junction, which is exacerbated with elevation of the arm above the shoulder. Robotic resection of the medial aspect of the first rib along with disarticulation of the costo-sternal joint has the best reported results to date.

Keywords: Thoracic Outlet Syndrome, TOS, Neurogenic, Venous TOS, Arterial TOS, Paget Schroetter Syndrome

In 1956, "Thoracic Outlet Syndrome" (TOS) was first proposed by Peet, a physical therapist at the Mayo Clinic, in order to unify the symptoms associated with many upper extremity neurovascular compression syndromes. [1] In 1958, Rob and Standeven used "Thoracic Outlet Compression Syndrome" in their description of a series of patients with cervical ribs, arterial thrombosis, and distal gangrene of the upper extremity. ${ }^{[2]}$ This attempt to unify a group of disparate anomalies that manifest with upper extremity neurovascular symptoms began an era which has been characterized by confusion about the underlying pathogenesis, confusion about the appropriate diagnostic tests, and poor therapeutic results.

\section{Historic Terms Used to Describe Upper Extremity Neurovascular Symptoms:}

Cervical Rib Syndrome- The first actual use of the term "cervical rib syndrome" is by $\mathrm{T}$. Wingate Todd in 1922. "Cervical rib syndrome" was the most well-known and widely used name for the syndrome throughout the late nineteenth century and into the early twentieth century. Although, recently, this term has fallen into disfavor, in reality, Cervical Rib Syndrome is an accurate description which should be reserved for neurovascular symptoms of the upper extremity caused by compression of the cervical ribs or the related anomalous bands on the brachial plexus or the extra thoracic portion of the subclavian artery in the neck. Cervical rib syndrome is rare within the group of disorders that result in upper extremity symptoms. Unfortunately, many who are not familiar with these conditions erroneously think that a cervical rib is responsible for thoracic outlet syndrome. The correct use of the term cervical rib syndrome plays an important role in the modern understanding of conditions that are responsible for upper extremity neurovascular symptoms.

Brachial Compression Neuritis- This term was first used by Stopford in 1919. " ${ }^{[4]}$ Although this is a reasonable attempt at describing compression 
of the brachial plexus in the neck, it is too general and does clearly convey the cause of such compression. Cervical rib syndrome is a more accurate description of what results in brachial compression neuritis.

\section{Scalenus Anticus Syndrome (Nafziger}

Syndrome)- "Scalenus anticus" is another name for the anterior scalene muscle. Adson and Coffey wrote a landmark paper in 1927 describing their approach of detaching the scalenus anticus from its insertion on the first rib in patients with a cervical rib. ${ }^{[5]}$ Ochsner, Gage, and DeBakey in 1935 were the first to describe patients with cervical rib syndrome without a cervical rib who had been treated successfully with the same procedure, ${ }^{[6]}$ They first used the term "scalenus anticus syndrome," but credited their work to Howard Naffziger and suggested the term "Naffziger syndrome" as well. Our present understanding of etiology of thoracic outlet syndrome would lead us to believe that although these authors accurately observed that there was compression of the subclavian vein at the medial aspect of the first rib, they erroneously attributed the compression to a hypertrophied anterior scalene muscle as opposed to a congenital abnormality of the first rib in the region of the insertion of the anterior scalene muscle, the anterior scalene tubercle.

\section{Costoclavicular Syndrome/ Costoclavicular} Compression Syndrome The mechanism of compression between the clavicle and the first rib was described by Eden in 1939 to explain vascular compression. ${ }^{[7]}$ Falconer and Weddell in 1943 described further cases with vascular compression. [8,9] The term "costoclavicular syndrome" was first used by Telford and Mottershead in 1947. ${ }^{[10]}$ It was observed that in patients with upper extremity neurovascular symptoms, the subclavian vein was compressed on the medial aspect of the first rib at its " $v$ " junction with the clavicle. In retrospect, this is a different interpretation of an observation of a phenomenon that was interpreted by other investigators as the scalenus anticus syndrome (Naffziger syndrome).

Hyperabduction Syndrome/Wright SyndromeThe term "hyperabduction syndrome" was first used by Beyer and Wright in 1951. [11] This descriptive term was based on the observations of Todd from 1913 to 1921, and Wright in 1945, who observed exacerbation of the upper extremity neurovascular symptoms with hyperabduction of the upper extremity above the shoulder. Once again, these instigators made similar observations in terms of compression of structures by a process in the medial aspect of the first rib. In addition, they emphasized that hyperabduction resulted in exacerbation of symptoms by further narrowing the space between the first rib and the clavicle. Brachiocephalic Syndrome/ Brachiocephalic Vascular Syndrome- In 1966, J.D. Devilliers first used the term "brachiocephalic vascular syndrome" to describe a young woman with a stroke related to arterial TOS and a cervical rib. [12]

Nocturnal Paresthetic Brachialgia/ Brachialgia Statica Paresthetica- This term was introduced by Wartenberg in 1944. [13] It refers to the same phenomenon as the hyperabduction syndrome/Wright syndrome.

Paget-Schroetter Syndrome/Effort Thrombosis of the Subclavian Vein/ Paget Schroetter Syndrome or Effort Thrombosis of the Subclavian Vein or Axillary-Subclavian Vein Thrombosis (ASVT) - In 1875, Sir James Paget described two patients with spontaneous 
swelling of one arm and prominence of veins over the ipsilateral chest. Paget thought the condition was caused by vasospasm. In 1884 , Leopold von Schroetter independently described patients with the same condition, and correctly attributed the clinical condition to the formation of occlusive venous thrombus. In 1949, Hughes performed a review of a large number of cases in the medical literature, and proposed the term, "Paget-Schroetter syndrome," which is still in use today. ${ }^{[14]}$ von Schroetter suspected that an episode of considerable exertion of the upper extremity precipitated the thrombosis. In 1942, Swartley first used the term, "effort thrombosis". [15] In Peet's attempt at consolidating all upper extremity neurovascular symptoms under thoracic outlet syndrome (TOS), PagetSchroetter syndrome became synonymous with Venous TOS.

Cervicobrachial SyndromeKenneth Aynesworth first used this term in a paper published in 1940, in which he described cervical rib syndrome under a different designation. ${ }^{[16]}$ It was used again by Hansson in 1941 , but since then the name has not come into common use. [17]

First Thoracic Rib Syndrome/Superior Outlet Syndrome/Fractured Clavicle Rib Syndrome/Cervical Rib and Band SyndromeThese miscellaneous names are of uncertain origin and have never come into common use.

Thoracic Inlet Syndrome- Specifically, thoracic inlet is the space defined by the opening into the chest cavity, which allows for the entry of vessels, the trachea, the esophagus, and other midline structures. Although some have tried to use this as a synonym for "thoracic outlet," clearly by convention, it relates to a different anatomic entity and should not be used in referring to compressive syndromes relating to patients with neurovascular symptoms of the upper extremity.

Pectoralis Minor Syndrome- In 1967, Erich Lang first referred to the "pectoralis minor syndrome". [18] This concept was built on a body of work by Jere Lord and Peter Stone in 1956, who performed the first documented pectoralis minor tenotomy in five patients diagnosed with hyperabduction syndrome. [19] Although this condition may be a rare cause of upper extremity neurovascular symptoms, regrettably in response to the poor surgical results with TOS, some modern surgeons have incorporated pectoralis minor tenotomy to procedures for TOS. Clearly, there is very little objective evidence for this practice.

Cervicodorsal Syndrome- First described by Nelson in 1957 in an attempt to simplify and group the various neurovascular syndromes of the upper extremity, this term has not come into common use. [20]

Cervicoaxillary Syndrome- In 1996, Ranney proposed this term to include subclassifcations "cervical outlet syndrome," "thoracic outlet syndrome," "costoclavicular syndrome," and "pectoralis minor syndrome," and to eliminate "thoracic inlet syndrome" [21]. This is yet a more recent attempt to group a number of etiologies resulting in upper extremity neurovascular symptoms. Such attempts at consolidation of disparate entities which have upper extremity neurovascular symptoms will most likely result in increasing the confusion which surrounds "TOS."

Thoracic Outlet Syndrome/Thoracic Outlet Compression Syndrome- In 1956, Peet proposed consolidation of all conditions with 
multitude of names and syndromes under "thoracic outlet syndrome" (TOS). In an attempt to clarify the various conditions that have been classified as TOS, investigators have described three types: neurogenic, venous, and arterial. Most investigators would agree that unification of multiple syndromes under a single umbrella term confuses the differences among closely related but different underlying pathological entities. It can only be concluded that the creation of the term, "thoracic outlet syndrome," was an unfortunate simplification, which is not consistent with the present understanding of the etiologic entities that are responsible for upper extremity neurovascular symptoms in patients. Given the confusion in terms of diagnosis and therapy that surrounds the diverse group of disorders that placed under the umbrella of thoracic outlet syndrome, future efforts should concentrate on separation of the different etiologic entities that result in neurovascular symptoms of the upper extremity. Results of surgery can only be improved through a clearer understanding of the specific etiologic entities and more directed surgical procedures which are designed to address the specific unequivocally diagnosed issues.

\section{Conventional Thinking about TOS:}

Since Peet's classification of TOS in 1956, conventionally TOS has been thought to represent a group of diverse disorders that result in compression of the neurovascular bundle exiting the thoracic outlet. The thoracic outlet has been defined as the triangular anatomical area between the clavicle and the first rib and the neck muscles which allows for the passage of the brachial plexus, subclavian artery, and subclavian vein. Compression of this area has been thought to result in a constellation of distinct symptoms, which can include upper extremity pallor, paresthesia, weakness, muscle atrophy, pain, and swelling. [22,23] Until recently, TOS classification has been based on symptoms, rather than the underlying pathology, with the subgroups consisting of neurogenic (NTOS), venous (VTOS or PSS), and arterial (ATOS) [85]. Neurogenic TOS accounts for over $95 \%$ of the cases, followed by venous (3-5\%) and arterial (1-2\%). [24] Neurogenic TOS (NTOS) has been further divided into True NTOS (TNTOS) and Disputed NTOS (DNTOS), with DNTOS reportedly representing $95-99 \%$ of all neurogenic cases. [25] The symptoms of TNTOS and DNTOS are largely the same, though objective findings from motor nerve conduction studies and needle electromyography are notably absent in the DNTOS. It has been reported that NTOS is more prevalent in women with a female to male ratio of 4:1. [26] TNTOS most commonly affects a younger woman in their teenage years and is usually unilateral. DNTOS is most commonly seen in women ranging from their 20 s to 60 s and is often bilateral. ${ }^{[25]}$ ATOS affects both genders equally and is mostly seen in young adults. ATOS is predominantly unilateral. VTOS, which presents as Paget-Schroetter syndrome, is more common in younger able-bodied individuals, most commonly affects the dominant upper extremity, and is associated with repetitive upper extremity activity. [26,27] The different demographics of these entities are an important clue to the etiology of these disease processes. This concept was ignored in Peet's classification of TOS.

\section{Historic Etiology of TOS:}

Peet's classification of TOS was based on the concept that symptoms were due to the 
compression of the neurovascular structures in the neck and upper chest. Accordingly, in searching for a hypothetical anatomic area where compression of the neurovascular structures occurs, three spaces were identified: interscalene triangle, costoclavicular space, and subcoracoid space. ${ }^{[25]}$ (Fig.1).

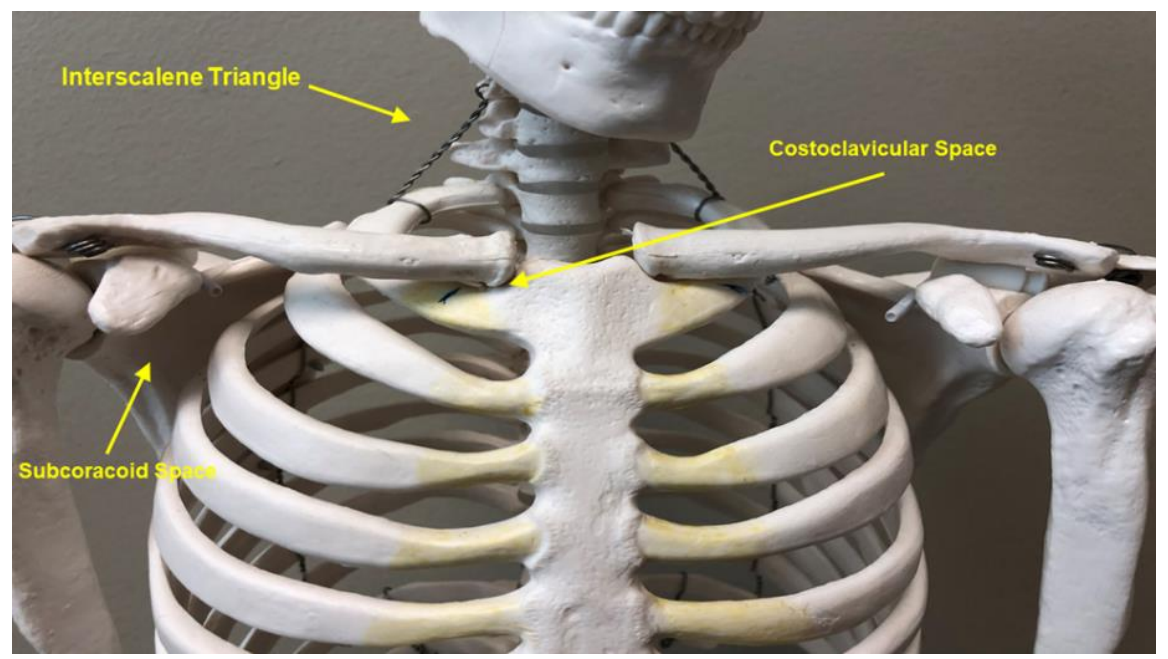

Figure 1. The interscalene triangle is created by the anterior scalene muscle anteriorly, middle scalene muscle posteriorly, and first rib inferiorly. The costoclavicular space is a ">" shaped space on the right and "<" shaped space on the left, at the junction of the frst rib, the sternum, and the clavicle. The subcoracoid space, the retropectoral space, or the subcoracoid pectoralis minor space is bordered anteriorly by pectoralis minor muscle, posteriorly by the chest wall, and superiorly by the coracoid process of the scapula.

The interscalene triangle is created by the anterior scalene muscle anteriorly, middle scalene muscle posteriorly, and first rib inferiorly. These structures create an elongated isosceles triangle. The brachial plexus passes through apex of this triangle in the neck and has no proximity to the first rib. On the other hand, the subclavian artery passes at the base of the triangle as its course over the first rib. The subclavian vein has no relationship to this triangle. The costoclavicular space is a ">" shaped space on the right and " $<$ " shaped space on the left, at the junction of the first rib, the sternum, and the clavicle. It is bordered by the costo-sternal joint medially, the anterior scalene muscle posterolaterally, and the first rib inferiorly at its base. The subclavian vein passes through this compartment, and the subclavian artery crosses the first rib just posterior to this compartment. The subclavian vein and artery are separated by the insertion of the anterior scalene onto the first rib. The subcoracoid space, the retropectoral space, or the subcoracoid pectoralis minor space is bordered anteriorly by pectoralis minor muscle, posteriorly by the chest wall, and superiorly by the coracoid process of the scapula. $[28,29]$ The brachial plexus passes through the subcoracoid space, and the subclavian artery and vein continue through it as the axillary artery and vein. The unifying characteristic of the classification of patients with neurovascular symptoms of the upper extremity into TOS by Peet and propagated in the past six decades has been "compression" of the neurovascular structures by an anatomic entity in the neck and upper 
chest. Proposed mechanism of compression has been the following:

Trauma: This includes midshaft clavicle fractures, whiplash injuries, or hemorrhage in the neck and upper chest. [25, 27]

Muscle hypertrophy and fibrosis: It has been hypothesized that repetitive activity results in fibrosis and eventual compression of nerves and vessels. VTOS or PSS has been attributed to this mechanism with thrombosis following repetitive motion of the upper extremity such as in athletes.

Anatomic anomalies: This includes Cervical Ribs and associated fibrous bands that originate from them and insert onto the first rib, or supernumerary scalene muscles. ${ }^{[30]}$

Benign or malignant tumors of the structures in the neck and upper chest. [31,32]

\section{Modern Understanding of TOS:}

Paget Schroetter Syndrome: Recently, examination of the medial aspect of the resected first ribs in patients with Paget-Schroetter syndrome has demonstrated the presence of a congenitally malformed bony tubercle that forms a tighter and wider joint at the junction of the first rib and the sternum. [33] In a number of patients with a wider tubercle, there is even a second pseudo-arthrosis with the head of the clavicle. $[34,35]$ It has been observed that the wider and less mobile cost-sternal joint "locks" the medial aspect of the first rib into place and results in extrinsic compression of the boney tubercle onto the subclavian vein at its junction with the innominate vein. Therefore, the abnormal medial aspect of the first rib compresses the subclavian vein at the "thoracic outlet." This abnormal tubercle on the medial aspect of the first rib can be seen on 3-D reconstruction of computerized axial tomograms in patients with PSS. (Fig. 2)

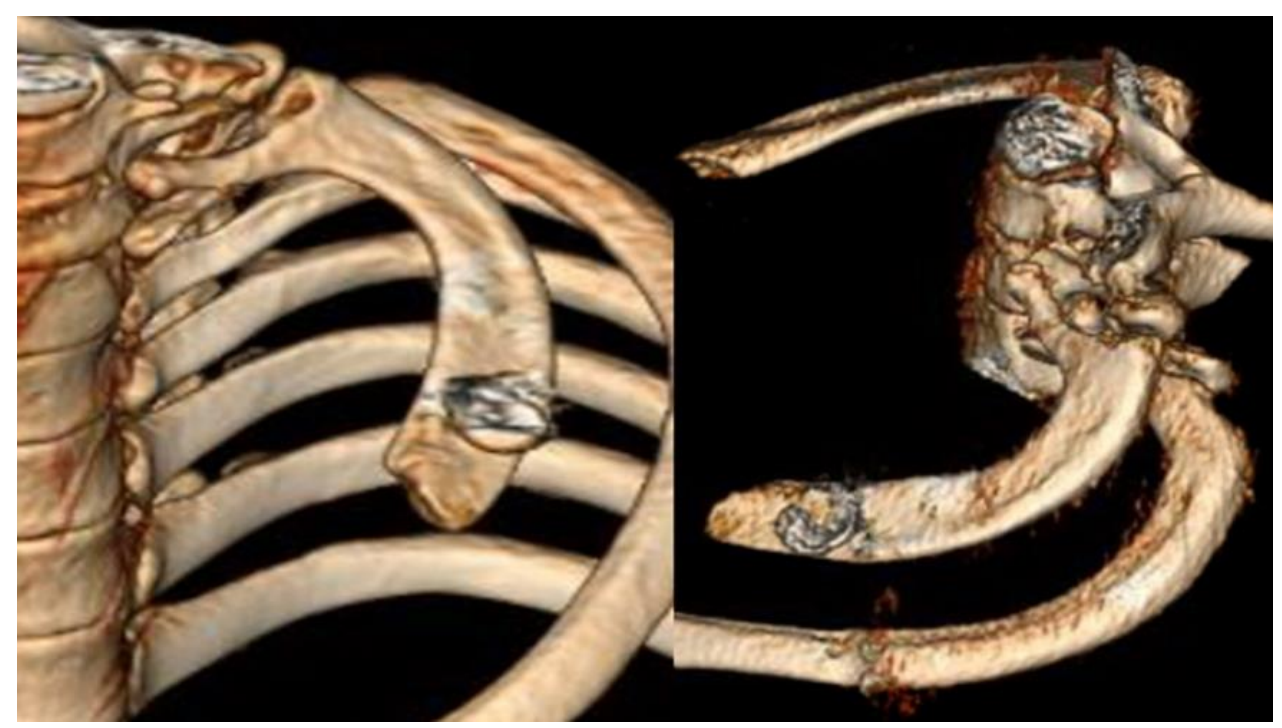

Figure 2. 3-D reconstruction of computerized axial tomograms in patients with Paget-Schroetter syndrome demonstrates the wider and less mobile cost-sternal joint that "locks" the medial aspect of the first rib into place and results in extrinsic compression of the bony tubercle onto the subclavian vein at its junction with the innominate vein.

In addition, the extrinsic compression of the dynamic magnetic resonance angiography subclavian vein by the tubercle at the medial aspect of the first rib can be demonstrated on

(MRA) with maneuvers and dynamic
venography. (Fig. 3) 


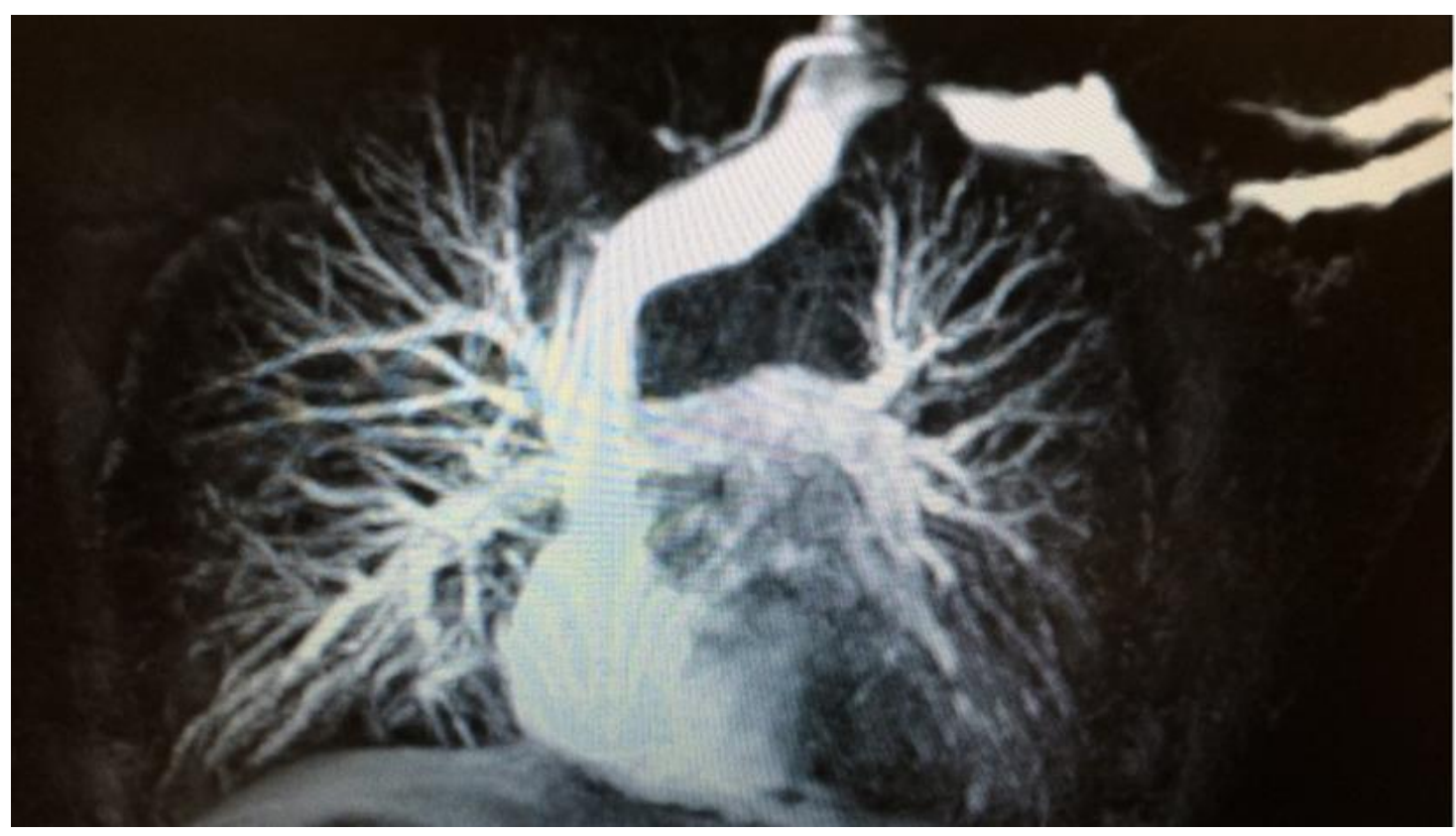

Figure 3. Magnetic resonance angiogram (MRA) with elevation of the left upper extremity shows compression of the subclavian vein by the abnormal bony tubercle in a patient with Paget-Schroetter Syndrome.

Furthermore, these studies clearly demonstrate that the subclavian vein compression increases with elevation of the arm above the shoulder. It can be surmised that without the benefit of the sophisticated modern imaging, and relying only on intraoperative observations, it is likely that historically surgeons have erroneously referred to this tubercle as the hypertrophied costoclavicular ligament and the hypertrophied scaleneus anticus tubercle. In patients with Paget- Schroetter syndrome, Gharagozloo et al. have demonstrated that disarticulation of the costo-sternal joint and resection of the offending portion of the first rib (portion of the rib medial to the subclavian artery) result in decompression of the subclavian vein. ${ }^{[34-38]}$ In addition, Dynamic Magnetic Resonance Angiography with arm maneuvers (MRA) in patients with PSS has demonstrated the presence of bilateral disease in the head of the first rib (Fig.4). This observation further clarifies the reports of Paget-Schroetter syndrome affecting the contralateral extremity and supports the congenital pathogenesis of the disease.

Therefore, it has been suggested that PagetSchroetter syndrome results from a congenital malformation of the first rib that compresses the subclavian vein at its junction with the innominate vein in the thoracic outlet, and with prolonged compression stemming from activities that elevate the arm above the shoulder, result in thrombosis of the vein.

Neurogenic TOS: Evaluation of patients with Neurogenic TOS who had persistent upper extremity pain following first rib resection by the transaxillary and supraclavicular approaches, revealed persistent extrinsic compression of the subclavian innominate junction on dynamic MRA. These patients underwent video assisted exploration of the chest, which showed a persistent costo-sternal joint despite evidence for prior removal of the remainder of the first rib. Disarticulation of the cost-sternal joint and removal of the remaining portion of the first rib, which bores a tubercle similar to that which was 
seen in patients with Paget- Schroetter syndrome, alleviated the extrinsic compression of the subclavian-innominate vein junction on postoperative dynamic MRA and resulted in relief of neurogenic symptoms in all patients. Based on this observation, it was hypothesized that neurogenic TOS may be the manifestation of nerve pain which results from venous compression, venous ischemia of the nerves in the upper extremity and the resultant diffuse neurologic symptoms. This hypothesis is based on the fact that the upper extremity is fed by a single artery and vein as an "end organ." In such a setting, studies have demonstrated that the blood-nerve barrier in the nerve root was more easily broken by venous congestion than by arterial ischemia. Venous congestion may be an essential factor precipitating circulatory disturbance in nerve roots and inducing neurogenic intermittent claudication. [39] Therefore, compression of the subclavian vein at its junction with the innominate vein may result in elevation of venous pressure, a decrease in arterial flow, and relative ischemia of the nerves of the upper extremity. Venous ischemia of the upper extremity nerves may manifest as pain, tingling, paresthesia and numbness, and varying degrees of neurogenic intermittent claudication depending on the degree and duration of venous compression. Elevation of the extremity above the shoulder may result in greater compression of the subclavian vein, further venous congestion, further decrease in arterial flow, greater degree of ischemia of the upper extremity nerves, and exacerbation of symptoms. This phenomenon is demonstrated on dynamic magnetic resonance imaging and venography. (Fig.5) The pathophysiology of nerve pain in this setting has been likened to symptoms that result from "crossing one leg over the knee."

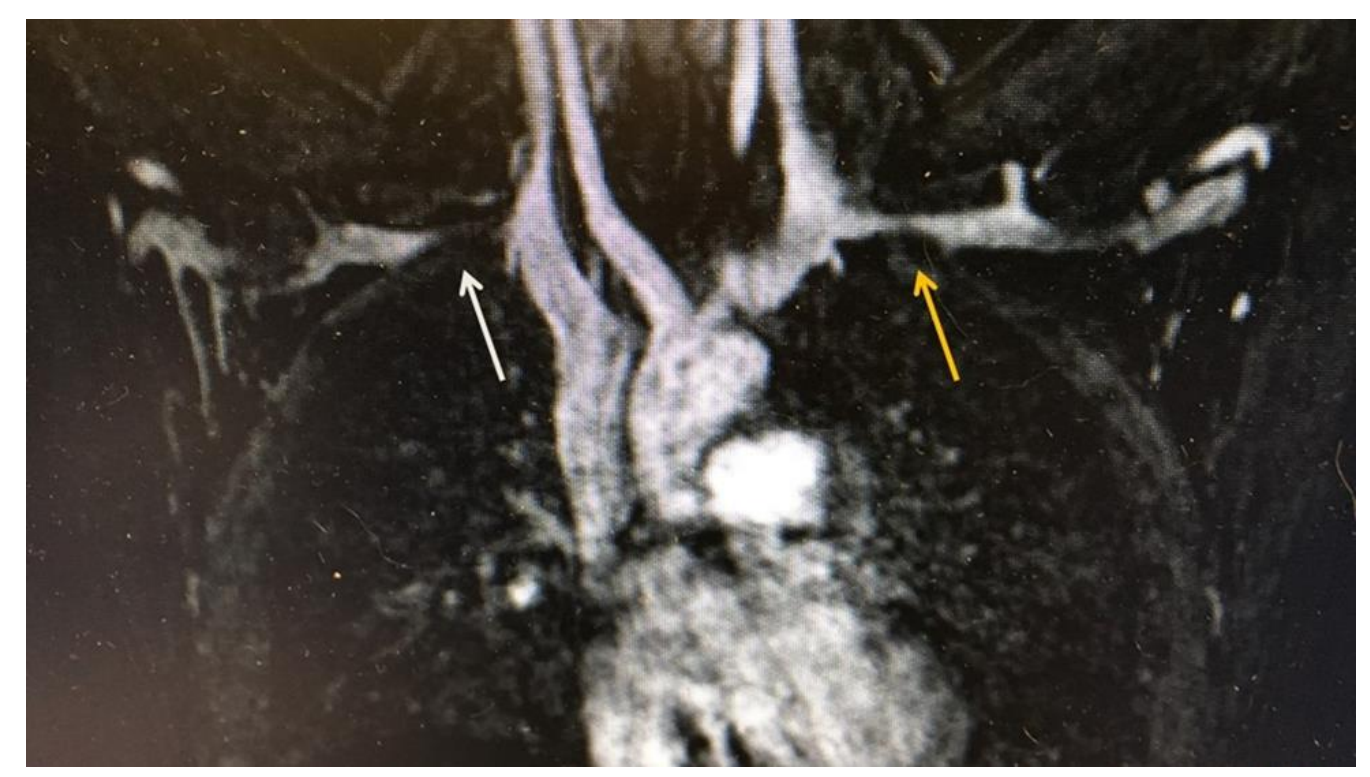

Figure 4. Magnetic resonance angiogram (MRA) with elevation of the left upper extremity in a patient who has had a transthoracic first rib resection on the right for "neurogenic" thoracic outlet syndrome. There is residual extrinsic compression (white arrow) of the right subclavian vein from the retained costo-sternal joint. In addition, the left subclavian vein also shows extrinsic compression at its junction with the innominate vein pointing to the bilateral and congenital nature of the disease (yellow arrow). 


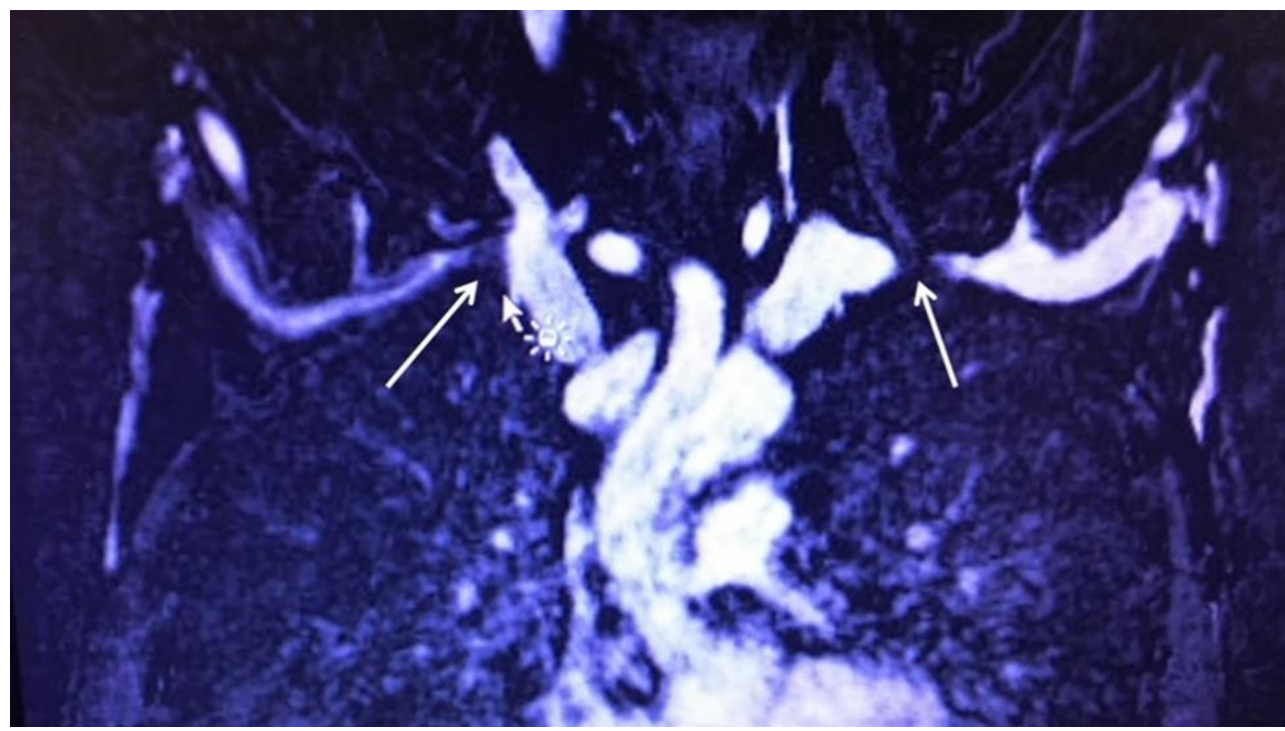

Figure 5. MRA in a patient with Disputed Neurogenic TOS. With elevation of the arms, there is bilateral compression (arrows) of the subclavian innominate vein junction.

Only $5 \%$ of patients with the diagnosis of neurogenic TOS are found to have a cervical rib or cervical bands and are best classified as cervical rib syndrome. Ninety-five percent of patients with the diagnosis of neurogenic TOS are believed to have neurologic manifestations of upper extremity ischemia and compression of the subclavian vein by an abnormal first rib at the thoracic outlet. Therefore, it was hypothesized that Robotic Transthoracic Resection of the Medial Aspect of the first rib at the costo-sternal junction in patients of neurogenic thoracic outlet syndrome diagnosed by MRA will result in relief of symptoms. In a proof-of-concept study, surgical outcomes in patients diagnosed with Disputed neurogenic thoracic outlet syndrome who underwent robotic first rib resection were reviewed. Diagnosis was made by history, physical exam, MRI of C-spine, orthopedic and neurologic examination, nerve conduction studies, and MRA of the thoracic outlet with maneuvers. Patients with cervical ribs or cervical bands were excluded.

Patients with compression of the subclavian vein by the medial aspect of the first rib underwent robotic resection of the first rib and were the subjects of this study. Subjective symptoms were assessed by Disabilities of the Arm, Shoulder and Hand Questionnaire Score (QuickDASH), at 1 week, 1 month, and 6 months. ${ }^{[38]}$ The extrinsic compression of the subclavian vein on postoperative MRA and angiogram with maneuvers was assessed at 1 month. In this proof of concept study, patients with neurologic symptoms of the upper extremity who were classified as Disputed "neurogenic TOS" had complete relief of symptoms with relief of compression of the subclavian vein after robotic resection of the medial aspect of the first rib and disarticulation of the costo-sternal joint. These observations have led these investigators to propose the "ischemic" versus the "compressive" mechanism for neurogenic TOS. They have likened neurogenic TOS in the upper extremity to the well-known phenomenon of paresthesia, numbness, and pain, which results from crossing one leg over the other at the knee. Despite commonly held belief that "Crossing Leg" syndrome results from compression of the peroneal nerve, it has been 
shown that in fact it is the result of the compression of the popliteal vein by the contralateral knee. The present understanding of TOS is that it is the manifestation of a congenital malformation of the first rib. The congenital malformation is in the form of a pronounced tubercle, which results in an abnormal costo-sternal joint and the compression of the subclavian vein at its junction with the innominate vein. The congenital disease is bilateral with variable symptomatic expression. The compression of the subclavian vein in the thoracic outlet results in a spectrum of disease which ranges from neurologic symptoms resulting from venous ischemia of the upper extremity nerves (Disputed Neurogenic TOS) to thrombosis of the subclavian vein with prolonged compression (Paget Schroetter or Venous TOS). Therefore, patients with "neurogenic Disputed "TOS and "venous" TOS or PSS represent two manifestation of the same disease process. This observation leads to the proposal that this type of thoracic outlet syndrome may be more accurately referred to as "Subclavian Vein Compression Syndrome."

\section{Rethinking the Etiology and Classification of "TOS"}

Peet's classifcation of TOS was based on symptomatic presentation of the disease. In the years that followed investigators tried to identify an anatomic cause. Based on recent studies, in order to decrease confusion and to improve therapeutic results with TOS, the disease should be classified based on the underlying pathologic entity. Acquired and traumatic abnormalities of the clavicle and first rib should be classified separately. Clearly after the more common and objectively supported diagnoses of conditions that result in neurovascular symptoms of the upper extremity, such as cervical spine disease, carpal tunnel disease, and nerve entrapment syndromes, have been ruled out, there remains a group of patients who are suspected of having TOS. In these patients, rather than the more usual classification such as arterial, venous, or neurogenic, the more accurate approach from a diagnostic and therapeutic approach is to classify them as:

Cervical Rib Disease: In these patients, an abnormal cervical rib or the associated bands that insert onto the first rib result in compression and displacement of the nerves or vessels in the neck. These patients can present with neurologic or vascular compromise. Patients with cervical rib syndrome (CRS) can have complications relating to compression of the subclavian artery and the brachial plexus secondary to a well-formed cervical rib, or to an incompletely formed first rib, fibrous band associated with a rudimentary cervical rib, or a giant transverse process of $\mathrm{C}$. Although in the past, these patients have been classified as TOS, separation of these patients into CRS allows for a more precise diagnostic and therapeutic strategy and perhaps more importantly clears the way to a better understanding of diseases that result from anomalies of the first rib.

\section{Thoracic Outlet Disease or "Subclavian Vein}

Compression Syndrome": In these patients an abnormal first rib at its junction with the sternum results in compression of the subclavian vein at the subclavian-innominate junction. Compression of the vein results in venous hypertension in the upper extremity and resultant neurologic symptoms. With prolonged compression of the subclavian-innominate junction, the vein clots giving rise to Paget- 
Schroetter syndrome. Therefore, patients who have been previously classified as Disputed Neurogenic and Venous TOS represent a variable symptomatic presentation of the same pathologic entity, which affects the subclavian vein. The term arterial TOS should be abandoned as these patients are better classified under cervical rib disease or under traumatic causes.

\section{Diagnosis of TOS:}

Historically confusion about the pathogenesis of TOS has resulted in a number of diagnostic tests. Conventionally diagnosis of TOS has been one of exclusion.

Patients presenting with upper extremity neurovascular symptoms need to undergo Physical Examination, Neck Radiograph, Ct of the Chest and Neck, Nerve Conduction Studies, MRI of the neck, MRA of the upper chest and venography.

As a general rule, except for angiography and MRA of the thoracic outlet with arm maneuvers, in diagnosing TOS, the other tests have been inconclusive and are of historic value. Although a great deal of emphasis has been placed on findings on physical exam, tests such as Adson's, Wright's, Roos, and Elvey are not diagnostic and should be avoided.

Emphasis should be placed on determining the presence of cervical abnormalities, and orthopedic anomalies that give rise to specific diagnoses.

In patients with Paget Schroetter Syndrome a venogram and MRA are diagnostic. After lysis of the thrombus, patients with PSS should undergo resection of the medial aspect of the first rib and disarticulation of the costo-sternal joint in order to relieve the extrinsic compression at the subclavian-innominate junction.
Patients with vague neurologic symptoms affecting the upper extremity, shoulder and neck, without specific neurologic findings have been classified as Disputed Neurogenic TOS. These patients should undergo an MRA with maneuvers. If extrinsic compression of the subclavian-innominate junction is demonstrated, these patients should undergo resection of the medial aspect of the first rib and disarticulation of the costo-sternal joint.

Presently MRA of the thoracic outlet with arm maneuvers is the test of choice in patients suspected of having TOS. This test shows the abnormal bony tubercle on the first rib with extrinsic compression of the subclavian innominate junction, which is exacerbated with elevation of the arm above the shoulder. The predictive value of surgical success of this test in patients with Paget-Schroetter presentation is $100 \%$, and in patients with Neurologic Presentation is $97.3 \%$. (38)

\section{Surgical Therapy of TOS:}

In 1910, Thomas Murphy, of Melbourne, Australia, performed the first resection of a normal first rib for the treatment of a patient who presented with upper extremity nerve-related symptoms without a visible cervical rib on X-ray. [40] The patient was a 28-year-old woman with symptoms of brachial plexus compression. Xrays showed no cervical rib, but Murphy was convinced the brachial plexus was compressed. Murphy performed surgery, finding the broad insertion of the anterior scalene was compressing the brachial plexus. He removed the insertion and a small part of the first rib. The patient had some but not complete relief in symptoms. In 1962, O.T. Clagett, in responding to the poor results associated with scalenectomy alone, proposed first rib resection through a high 
posterior thoracotomy. [41] Results of first rib resection with this approach were confusing as the preoperative upper extremity morbidity could not be differentiated from postoperative morbidity. On one hand, patients presented with upper extremity symptoms preoperatively, but on the other hand, the very invasive surgical procedure resulted in additional upper extremity morbidity following surgery. In 1966, in seeking to obviate the morbidity associated with Clagett's high posterior thoracotomy approach, David Roos described a transaxillary approach to first rib resection. [42] However, in the experience of most surgeons, the Roos transaxillary approach is associated with high rates of neurovascular complications, erroneous resection of the second rib, and overall poor relief of symptoms. ${ }^{[43,44]}$ In response to the inconsistent results to first rib resection, in 1972, Silver and Vemuri attributed some of the failures after first rib resection to the compression of the brachial plexus in the retro-pectoral space and suggested pectoralis minor tenotomy, either in isolation or in addition to first rib resection. ${ }^{445,46]}$ This was a rediscovery of a technique that was advocated two decades earlier by Lord and Stone for "effort thrombosis" and "hyper abduction syndrome" with inconsistent results. [47] In 1979, Sanders introduced supraclavicular scalenectomy in patients with recurrent symptoms following first rib resection for posttraumatic upper extremity symptoms. Scalenectomy was later used as the primary treatment in patients with upper extremity symptoms following neck trauma [48]. Unfortunately, as illustrated by the history of TOS, as the result of the ongoing confusion and the poor and inconsistent surgical results, surgeons have erroneously advocated scalenectomy with or without resection of the first rib for patients without neck trauma. In the near six decades since Peet's report, confusion about TOS and the poor results with surgical intervention have continued. During this time, a great deal of attention has been focused on the surgical approach to the resection of the first rib and whether other procedures such as scalenectomy, neurolysis, and pectoralis minor tenotomy should be included with first rib resection. However, irrespective of the approach and the specifics of the surgical procedures, the results of conventional surgery have been disappointing. ${ }^{449,50]}$

Due to the confusion about the etiology of TOS and the poor surgical results, the treatment of patients with Disputed NTOS has been very controversial. As a result, in these patients, conservative management is recommended as the first line of therapy. Although there has been no consensus as to the appropriate conservative regimen, it has included patient education, postural mechanics, weight control, relaxation techniques, activity modification, active stretching, targeted muscle strengthening, TOSspecific rehabilitation, and pharmacologic therapies. ${ }^{[51]}$ Pharmacologic interventions have included NSAIDs and/or opioid analgesics, muscle relaxants, anticonvulsants, antidepressants, and injection of local anesthetic, steroids, or botulinum toxin type $A$ into the anterior scalene. Invariably conservative management has been associated with suboptimal results and poor patient satisfaction. $[52,53]$ First rib resection with the hypothesized outcome of decompressing the thoracic outlet has been recommended in patients with NTOS who fail conservative management, even 
without a specific diagnosis of the etiologic entity.

\section{Modern Strategy for the Surgical Treatment of TOS:}

Treatment of ATOS and VTOS is not controversial. ATOS is usually the result of compression of the subclavian artery by an abnormality in the cervical region most often associated with cervical rib syndrome. In these patients, the optimal therapeutic regimen includes initial thrombolysis and anticoagulation followed by removal of the cervical rib or bands that result in compression.

Management of VTOS or PSS consists of thrombolytic therapy and anticoagulation, followed by resection of the first rib. It is crucial to disarticulate the costo-sternal joint as the pathologic entity, the abnormal boney tubercle is part of the joint. Simply transecting the rib proximal to the joint will not relieve the compression at the subclavian-innominate junction and result in what has been called "recurrent" disease.

In patients with vague neurologic symptoms which consist of pain, and paresthesia affecting the arm, shoulder and the neck, who have heretofore been classified as Disputed NTOS, an MRA with maneuvers provides definitive diagnosis. In these patients, disarticulation of the first rib from the sternum at the costo-sternal joint and removal of the rib medial to the subclavian artery has been shown to relieve the symptoms.

Surgical approaches to resection of the first rib have included transthoracic, transaxillary, supraclavicular, infraclavicular, and

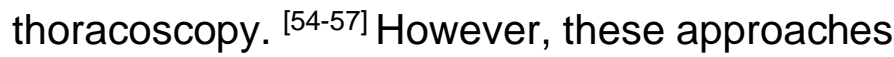
are associated with neurovascular complications, incomplete decompression of the subclavian vein and the medial aspect of the thoracic outlet, and incomplete resection of the most medial portion of the rib. Brachial plexus injury after transaxillary frst rib resection has ranged from 0.6 to $9 \%$, and $4 \%$ after supraclavicular first rib resection. [58,59] These complications are in large part the result of the extrathoracic surgical approach and the difficulty with exposure and access. In addition, the concept has been to remove the body of the first rib and leave the joints at the two ends of the rib intact. This concept has been reinforced by the technical difficulty of disarticulating the costosternal joint by a transthoracic, supraclavicular, or VATS approach. Ironically, based on the present understanding of the "offending" portion of the first rib which is located at the costo-sternal joint, transecting the rib at the joint and removal of the rib is ineffective in relieving the extrinsic compression of the subclavian-innominate vein junction. Indeed, this observation may be the explanation for the lack of relief of neurologic symptoms in a significant number of patients with "neurogenic" TOS, and failure to attain full patency of the subclavian vein in patients with "venous" TOS. Theoretically, a minimally invasive transthoracic approach aimed at removing the offending compressive portion of the first rib would obviate the neurovascular complications and allow for complete resection of the offending portion of the first rib. The robotic surgical systems have the advantages of 3D highdefinition visualization, precise instrument maneuverability in a confined space. The technique of robotic first rib resection has been described previously. (35) (Figs.6,7) 


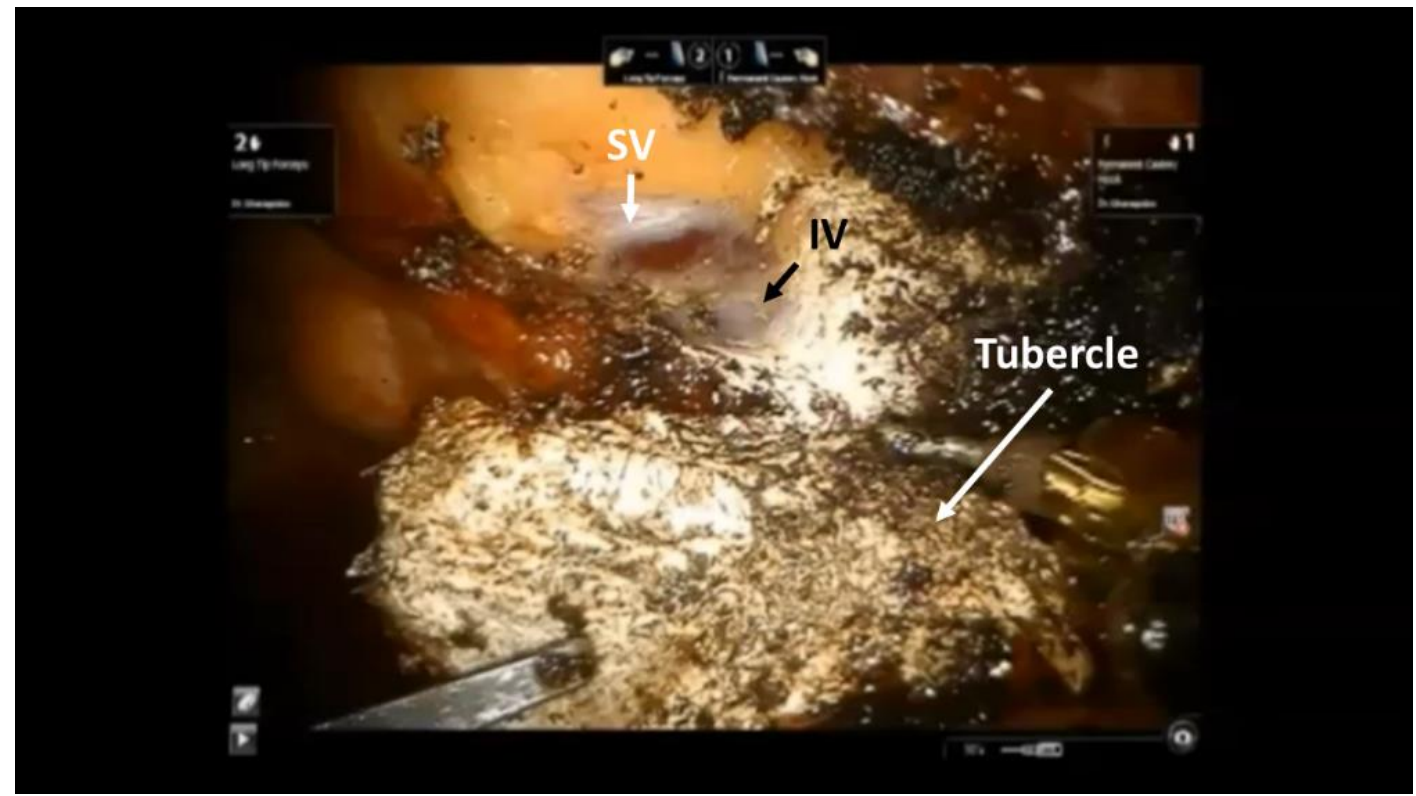

Figure 6. Intraoperative photograph during the robotic resection of the medial right first rib in a patient with Disputed Neurogenic TOS. The abnormal boney tubercle at the costo-steral joint results in compression of the subclavian vein (SV) at its junction with the innominate vein (IV).

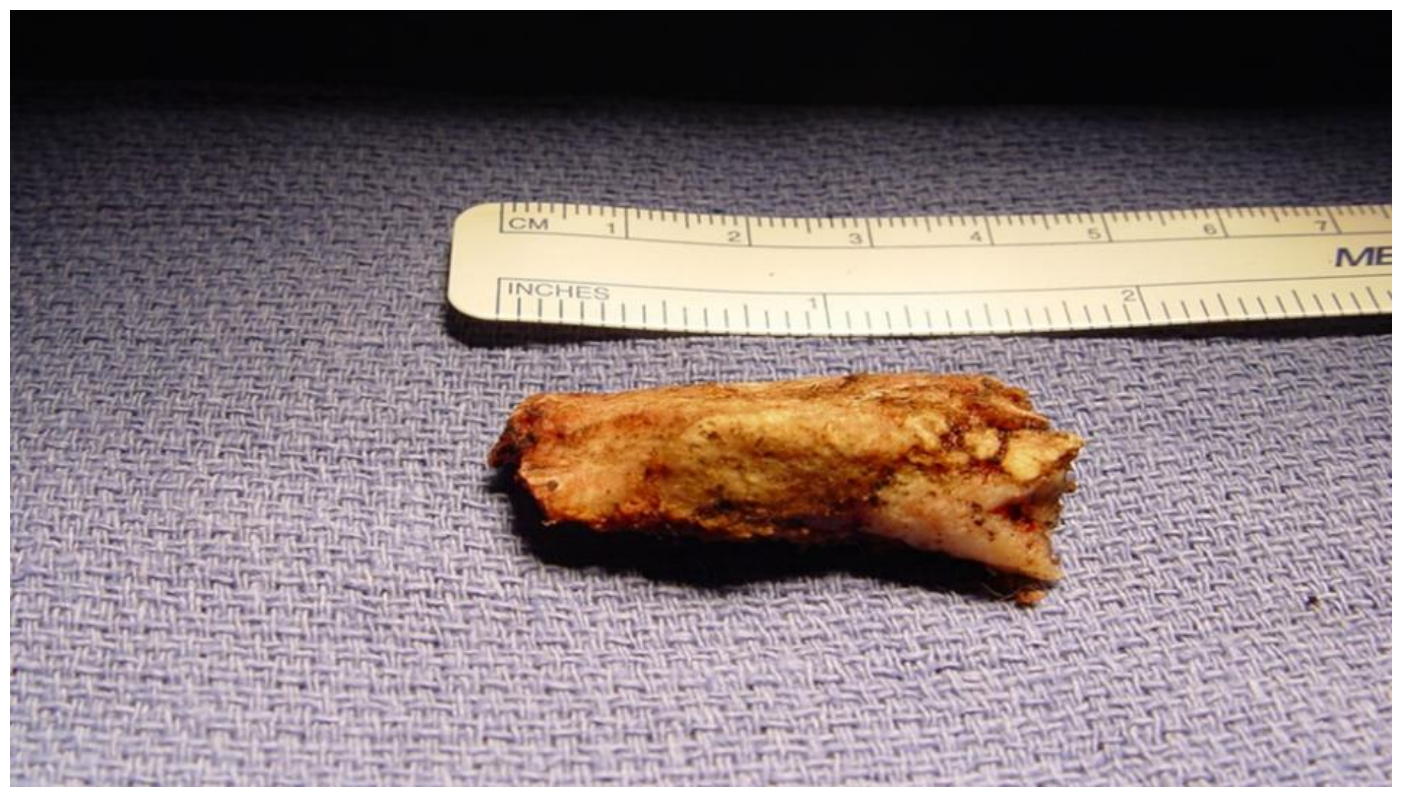

Figure 7. The resected specimen of the "offending" portion of the first rib from a patient with Disputed Neurogenic TOS syndrome showing the abnormal bony tubercle on the medial aspect of the first rib that compresses the subclavian vein at the subclavian-innominate junction.

The results of this technique have been the best reported to date. (38) Gharagozloo et. al. reported a total of 162 patients. Seventy-nine patients underwent robotic resection of the offending portion of the first rib for neurologic symptoms of the upper extremity (Disputed neurogenic TOS). There were 29 men, 50 women. Mean age was $34+/-9.5$ years. Eightythree patients underwent transthoracic robotic first rib resection for Paget-Schroetter syndrome. There were 49 men and 34 women. Mean age was $24+/-8.5$ years. Extrinsic compression of the subclavian vein by the medial aspect of the first rib was demonstrated 
on MRA with maneuvers in all patients. The first rib medial to the subclavian artery was removed en bloc and the costo-sternal joint was disarticulated. In all patients, a bony protuberance articulated with sternum. Operative time was 87.6 minutes +/10.8 minutes. There were no intraoperative complications. Hospital stay ranged from 2 to 4 days with a median hospitalization of 3 days. There were no neurovascular complications. There was no mortality. In patients with neurologic symptoms, Quick DASH Scores (Mean +/- SEM) decreased from 60.3+/2.1 preoperatively to $5+/-2.3$ in the immediate postoperative period, and $3.5+/ 1.1$ at 6 months. At 3,6,12, and 24 months, in all patients, MRA with maneuvers showed relief of extrinsic compression and patency of the subclavian vein. In patients with PSS, two years after robotic resection of the offending portion of the first rib and obtaining patency of the subclavian vein, all patients remained asymptomatic and had full function of the affected upper extremity.

\section{References}

[1] Peet RM. Thoracic-outlet syndrome: evaluation of a therapeutic exercise program. Proc Staff Meet Mayo Clinic. 1956;31:281.

[2] Rob CG, Standeven A. Arterial occlusion complicating thoracic outlet compression syndrome. $\mathrm{Br}$ Med J. 1958;2:709-12.

[3] Werden S. Radiologic imaging in diagnosis and assessment of NTOS. In: Illig KA, Thompson RW, Freischlag J, Donohue DM, Edgelow PI, Jordan SE, eds. Thoracic outlet syndrome.1st edn. New York: Springer. pp.111-25.

[4] Stopford JSB, Telford ED. Compression of the lower trunk of the brachial plexus by a first dorsal rib. Brit J Surg. 1919;7:168.
[5] Adson WA. Surgical treatment for symptoms produced by cervical ribs and the scalenus anticus muscle. Surg Gynecol Obstet. 1947;85:687-700.

[6] Ochsner A, Gage M, Debakey M. Scalenus anticus syndrome. Am J Surg. 1935;28:669-95.

[7] Eden KC. The vascular complications of cervical ribs and frst thoracic rib abnormalities. $\mathrm{Br} \mathrm{J}$ Surg. 1939;27:111-39

[8] Falconer MA, Weddell G. Costoclavicular compression of the subclavian artery and vein. Lancet. 1943;2:539-43.

[9] Falconer MA, Li FW. Resection of the first rib in costoclavicular compression of the brachial plexus. Lancet. 1962 Jan 13;1:59-63.

[10]Bramwell E, Dykes HB. Rib pressure and the brachial plexus. Edinburgh Med J. 1927;27:65.

[11]Beyer JA. The hyperabduction syndrome, with special reference to its relationship to Raynaud's syndrome. Circulation. 1951;4:161-72

[12] De Villiers JC. A brachiocephalic vascular syndrome associated with cervical rib. $\mathrm{Br}$ Med $\mathrm{J}$. 1966;2:140-3.

[13] Wartenberg R. Brachialgia Statica Paresthetica. J Nerv Ment Dis. 1944;5:877.

[14]Hughes ES. Venous obstruction in the upper extremity; PagetSchroetter's syndrome; a review of 320 cases. Surg Gynecol Obstet. 1949;88:89-127.

[15] Swartley WB, Weeder SD, McLaughlin EF. Thrombosis and gangrene of the right arm associated with polycythemia vera: its relation to effort thrombosis. Ann Surg. 1942;116:184-93

[16] Aynesworth KH. The Cervicobrachial syn drome. Ann of Surg. 1940;5:724-42.

[17] Hanson KG. The Cervicobrachial syndrome. Arch Phys Ther. 1941;22:662-6

[18]Lang EK. Scalinus anticus and pectoris minor syndrome. J Indiana Med Assoc. 1967;80:440.

[19] Lord JR, Stone PW. Pectoralis minor Tenotomy and anterior Scalenotomy with special reference to the Hyperabduction syndrome and "effort AJSRR: https://escipub.com/american-journal-of-surgical-research-and-reviews/ 16 
thrombosis" of the subclavian vein. Circulation. 1956;13: 537-42.

[20]Nelson PA. Treatment of patients with cervicodorsal outlet syndrome. J Am Med Assoc. 1957; 163:1570-6.

[21] Ranney D. Thoracic outlet: an anatomical redefnition that makes clinical sense. Clin Anatomy. 1996;9:50-2.

[22] Laulan J, Fouquet $B$, Rodaix $C$, Jauffret $P$, Roquelaure $\mathrm{Y}$, Descatha $\mathrm{A}$. Thoracic outlet syndrome: defnition, aetiological factors, diagnosis, management and occupational impact. J Occup Rehabil. 2011;21(3):366-73.

[23] Jones MR, Prabhakar A, Viswanath O, Urits I, Green JB, Kendrick JB, Brunk AJ, Eng MR, Orhurhu V, Cornett EM, Kaye AD. Thoracic outlet syndrome: a comprehensive review of pathophysiology, diagnosis, and treatment. Pain Ther. 2019; 8:5-18

[24]Freischlag J, Orion K. Understanding thoracic outlet syndrome. Scientifca (Cairo). 2014;2014:16

[25] Stewman C, Vitanzo PC, Harwood MI. Neurologic thoracic outlet syndrome: summarizing a complex history and evolution. Curr Sports Med Rep. 2014;13(2):100-6.

[26] Ferrante MA, Ferrante ND. The thoracic outlet syndromes: part 2. The arterial, venous, neurovascular, and disputed thoracic outlet syndromes. Muscle Nerve. 2017;56(4):663-73.

[27]Brooke BS, Freischlag JA. Contemporary management of thoracic outlet syndrome. Curr Opin Cardiol. 2010;25(6):535-40

[28] Raptis CA, Sridhar S, Thompson RW, Fowler KJ, Bhalla S. Imaging of the patient with thoracic outlet syndrome. Radiographics. 2016;36(4):9841000.

[29]Ferrante MA. The thoracic outlet syndromes. Muscle Nerve. 2012;45(6):780-95.
[30] Sanders RJ, Hammond SL, Rao NM. Diagnosis of thoracic outlet syndrome. J Vasc Surg. 2007;46 (3):601-4.

[31] Davis GA, Knight SR. Pancoast tumors. Neurosurg Clin N Am. 2008;19(4):545-57.

[32] Abdolrazaghi $H$, Riyahi A, Taghavi M, Farshidmehr $P$, Mohammadbeigi A. Concomitant neurogenic and vascular thoracic outlet syndrome due to multiple exostoses. Ann Card Anaesth. 2018;21 (1):71-3.

[33] Gharagozloo F, Meyer M, Tempesta B, et al. Proposed pathogenesis of Paget-Shroetter disease: impingement of the subclavian vein by a congenitally malformed boney tubercle on the first rib. J Clin Pathol. 2012;65:262-6

[34] Gharagozloo F, Meyer M, Tempesta B, et al. Robotic en bloc frst-rib resection for PagetSchroetter disease, a form of thoracic outlet syndrome: technique and initial results. Innovations. 2012; 7(1):39-44.

[35] Gharagozloo F, Meyer M, Tempesta B, et al. Robotic transthoracic first rib resection for PagetSchroetter syndrome. Eur J Cardiothorac Surg. 2019;55:434-9

[36] Gharagozloo F, Meyer M, Tempesta B, Werden S. Robotic First Rib Resection.

[37]Gharagozloo F, Atiquzzaman N, Meyer $M$, Tempesta B, Werden S. Robotic first rib resection for thoracic outlet syndrome. J Thorac Dis 2020 | http://dx.doi.org/10.21037/jtd-2019-rts-04.

[38] Gharagozloo F, Tempest ab, Weden S, Meyer M. Robotic First Rib Resection for Thoracic Outlet Syndrome. Surg Tech Int. 2020; 36:1228.

[39] Kobayashi S, Takeno K, Miyazaki T, et al. Effects of arterial ischemia and venous congestion on the lumbar nerve root in dogs. J Orthop Res. 2008;26(11):1533-40

[40] Murphy T. Brachial plexus neuritis caused by pressure of the first rib. Aust Med J. 1910;15:5825.

AJSRR: https://escipub.com/american-journal-of-surgical-research-and-reviews/ 
[41]Clagett OT. Presidential address, American Association of Thoracic Surgery Research and Prosearch. J Thorac Cardiovasc Surg. 1962;44: 153-66.

[42] Roos DB. Transaxillary approach for frst rib resection to relieve thoracic outlet syndrome. Ann Surg. 1966;163:354-8.

[43] Urschel HC. Management of the thoracic-outlet syndrome. N Engl J Med. 1972;286:1140-3.

[44] Sanders RJ, Monsour JW, Baer SB. Transaxillary first rib resection for the thoracic outlet syndrome. Arch Surg. 1968;97(6):1014-23.

[45] Vemuri C, Wittenberg AM, Caputo FJ, Earley JA, Driskill MR, Rastogi R, Emery VB, Thompson RW. Early effectiveness of isolated pectoralis minor tenotomy in selected patients with neurogenic thoracic outlet syndrome. J Vasc Surg. 2013;57: 1345-52.

[46] Silver D. Thoracic outlet syndrome. In: Textbook of Surgery (Davis: Christopher). 10th ed. Philadel phia: W. B. Saunders Co; 1972. p. 1858.

[47] Lord JR, Stone PW. Pectoralis minor Tenotomy and anterior Scalenotomy with special reference to the Hyperabduction syndrome and "effort thrombosis" of the subclavian vein. Circulation. 1956; 13:537-42

[48] Sanders RJ, Monsour JW, Gerber WF, Adams WR, Thompson N. Scalenectomy versus first rib resection for treatment of the thoracic outlet syndrome. Surgery. 1979;85:109-21

[49]Peek J, Vos CG, Ünlü Ç, et al. Long-term functional outcome of surgical treatment for thoracic outlet syndrome. Diagnostics (Basel). 2018;8(1): 12.

[50] Peek J, Vos CG, Ünlü Ç, et al. Outcome of surgical treatment for thoracic outlet syndrome: systematic review and meta-analysis. Ann Vasc Surg 2017;40:303-326.

[51] Huang JH, Zager EL. Thoracic outlet syndrome. Neurosurgery. 2004;55(4):897-902.

[52] Foley JM, Finlayson $H$, Travlos A. A review of thoracic outlet syndrome and the possible role of botulinum toxin in the treatment of this syndrome. Toxins (Basel). 2012;4(11):1223-35.

[53] Finlayson HC, O'Connor RJ, Brasher PMA, Travlos A. Botulinum toxin injection for management of thoracic outlet syndrome: a double-blind, randomized, controlled trial. Pain. 2011;152(9):2023-8

[54] Weaver M, Lum Y. New diagnostic and treatment modalities for neurogenic thoracic outlet syndrome. Diagnostics. 2017;7(2):28.

[55] Hwang J, Min BJ, Jo W-M, Shin JS. Video-assisted thoracoscopic surgery for intrathoracic frst rib resection in thoracic outlet syndrome. J Thorac Dis. 2017;9(7):2022-8.

[56] George RS, Milton R, Chaudhuri N, et al. Totally endoscopic (VATS) frst rib resection for thoracic outlet syndrome. Ann Thorac Surg. 2017;103:2415

[57] Ohtsuka T, Wolf RK, Dunsker SB. Port-access first-rib resection. Surg Endosc. 1999;13:940-2.

[58] Chang DC, Lidor AO, Matsen SL, Freischlag JA. Reported inihospital complications following rib resections for neurogenic thoracic outlet syn drome. Ann Vasc Surg. 2007;21(5):564-70.

[59]Hosseinian MA, Loron AG, Soleimanifard Y. Evaluation of complications after surgical treatment of thoracic outlet syndrome. Korean J Thorac Cardiovasc Surg. 2017;50(1):36-40. 
Farid Gharagozloo, M.D. et al., AJSRR, 2021; 4:27 